\title{
Lung B cells promote early pathogen dissemination and hasten death from inhalation anthrax
}

\author{
M Rayamajhi ${ }^{1,2}$, C Delgado $^{1,2}$, TV Condon ${ }^{1,2}$, DW Riches $^{1-3}$ and LL Lenz ${ }^{1,2}$
}

Sampling of mucosal antigens regulates immune responses but may also promote dissemination of mucosal pathogens. Lung dendritic cells (LDCs) capture antigens and traffic them to lung-draining lymph nodes (LDLNs) dependent on the chemokine receptor CCR7 (chemokine (C-C motif) receptor 7 ). LDCs also capture lung pathogens such as Bacillus anthracis (BA). However, we show here that the initial traffic of BA spores from lungs to LDLNs is largely independent of LDCs and CCR7, occurring instead in association with $B$ cells. BA spores rapidly bound $B$ cells in lungs and cultured mouse and human $B$ cells. Binding was independent of the $B$-cell receptor (BCR). $B$ cells instilled in the lungs trafficked to LDLNs and BA spore traffic to LDLNs was impaired by B-cell deficiency. Depletion of B cells also delayed death of mice receiving a lethal $B A$ infection. These results suggest that mucosal $B$ cells traffic $B A$, and possibly other antigens, from lungs to LDLNs.

\section{INTRODUCTION}

Mucosal epithelia serve as barriers to the establishment of systemic bacterial infections. However, many pathogenic bacteria have evolved virulence strategies that enable them to overcome these barriers, disseminate from mucosa to peripheral tissues, and initiate systemic disease. ${ }^{1}$ One mechanism for systemic dissemination of bacteria involves their phagocytosis and trafficking by host dendritic cells (DCs). ${ }^{2,3}$ However, pathogen trafficking to peripheral lymph nodes (LNs) can also occur via DC-independent mechanisms. ${ }^{4,5}$ It is not clear how such DC-independent dissemination of pathogenic bacteria occurs, or whether it contributes to host susceptibility.

DC migration from peripheral tissues to draining lymphoid tissues occurs when the DCs receive an activation signal, for example, from inflammatory stimuli. ${ }^{6}$ Activated DCs upregulate expression of the LN-homing chemokine receptor CCR7 (chemokine (C-C motif) receptor 7), which mediates their traffic to draining lymphatics. ${ }^{7}$ CCR7 is critical for migration of skin DCs to draining lymphatics, ${ }^{7}$ migration of intestinal DCs from the lamina propria to mesenteric LNs, ${ }^{7,8}$ and for migration of lung DCs (LDCs) from lung alveolae to lung-draining LNs (LDLNs). ${ }^{9}$ However, CCR7 is not required for the entry of $\mathrm{B}$ and T cells into LNs. ${ }^{10}$
Bacillus anthracis (BA) is a spore-forming bacterium that infects diverse mucosal tissues. Pulmonary exposure to BA spores causes inhalation anthrax, a rare but highly fatal disease. Data from humans contracting anthrax and from animal models consistently indicate that BA spores disseminate from the lungs to LDLNs within $5 \mathrm{~h}$ of their inhalation. ${ }^{11-13}$ It is thought that this rapid dissemination is responsible for the development of lethal systemic disease. In the lung, BA spores are rapidly engulfed by immune cells such as phagocytic LDCs and alveolar macrophages (AMs). ${ }^{14}$ It has been suggested that LDCs traffic phagocytosed BA spores away from the lungs. ${ }^{15}$ However, trafficking of LDCs to the LDLNs reportedly peaks at $24 \mathrm{~h}$ after antigen or pathogen exposure. ${ }^{9} 16$ Thus, it is not clear whether BA spore trafficking by LDCs is responsible for the very rapid initial spore dissemination. It is also unknown whether trafficking of spores by LDCs is required for establishment of systemic BA infection.

In addition to LDC and AMs, the lungs of mice and humans contain a third population of professional antigen-presenting cells: B cells. Lung B cells are essential for maintaining pulmonary homeostasis and actively contribute to immune responses against a variety of pathogens. ${ }^{17,18} \mathrm{~B}$ cells can respond to pathogens through their cell surface B-cell immunoglobulin receptor

${ }^{1}$ Integrated Department of Immunology, National Jewish Health, Denver, Colorado, USA. ${ }^{2}$ Integrated Department of Immunology, University of Colorado, Denver, Denver, Colorado, USA. ${ }^{3}$ Program in Cell Biology, Department of Pediatrics, National Jewish Health, Denver, Colorado, USA. Correspondence: LL Lenz (lenzl@njhealth.org) 
(BCR) as well as a variety of innate receptors that recognize microbial products independent of the BCR. ${ }^{19}$ Indeed, B cells have been shown to influence early immune responses to Mycobacterium tuberculosis ${ }^{20}$ and Salmonella typhimurium ${ }^{21}$ independent of antibody production.

Here, we employed a mouse model of inhalation anthrax with spores of the attenuated Sterne BA strain to investigate the mechanisms of BA spore traffic from the lungs to the LDLNs. Using both Sterne-resistant C57BL/6 and Sterne-sensitive A/J mouse strains, we confirmed previous reports that BA spores traffic to the LDLNs within a few hours of exposure. We further showed that such trafficking was independent of LDCs and CCR7 expression. Instead, BA spores rapidly associated with $B$ cells in the lungs of infected mice and soon thereafter were observed in association with $B$ cells in the LDLNs. We observed migration of B cells from the lung to the LDLNs and found that depletion of B cells from the lungs impaired the initial trafficking of spores to the LDLNs. B-cell depletion also significantly delayed death of lethally infected $\mathrm{A} / \mathrm{J}$ mice. These findings suggest that $\mathrm{B}$ cells contribute to the DC-independent establishment of systemic infections by mucosal pathogens.

\section{RESULTS}

\section{Rapid traffic of BA spores from the lungs to the LDLNs}

To study trafficking of BA spores following pulmonary in vivo infection, we fluorescently labeled highly purified spores of Sterne (34F2) strain BA (pXO1+X02-) using DyLight 649 and administered them to Sterne-susceptible A/J mice using a nonsurgical intratracheal (i.t.) inoculation procedure. ${ }^{22}$ The labeled spores were uniformly bright and readily detected using flow cytometry (Figure 1a). When the labeled spores were administered to $\mathrm{A} / \mathrm{J}$ mice, we reproducibly detected a small population of DyLight $649+$ host cells in the LDLNs within $6 \mathrm{~h}$ (Figure 1b). These data indicated that transport of BA spores to the LDLNs occurs rapidly and reproducibly in this model infection. Comparison of DyLight 649 + cell numbers from the LDLNs with numbers of heatresistant colony-forming units (CFUs) obtained from lysates of LDLNs revealed an average ratio of $0.35 \pm 0.01$ across three independent experiments (Figure 1c). Thus, the number of DyLight $649+$ cells consistently corresponded to approximately one-third of the number of live spores. This suggests that each DyLight $649+$ cell carried an average of $\sim 3$ viable spores. Hereafter, we refer to DyLight $649+$ cells as spore + cells.

Spore + cells accumulated in the LDLNs as early as $30 \mathrm{~min}$ after i.t. inoculation with $10^{8}$ DyLight $649+$ spores (Figure 1d). The number of spore + cells detected in the LDLNs increased to $\sim 0.015 \%$ of the total cells by 5 hours post injection (h.p.i.; Figure 1b). No DyLight $649+$ cells or BA CFUs were observed in the spleens or blood of infected animals at these early time points. These data are consistent with prior histological observations of virulent BA spores in LDLNs within 5 h.p.i., ${ }^{11,13,15}$ and suggest that BA spores are rapidly and specifically trafficked from lungs to the LDLN.

\section{CCR7 is dispensable for trafficking of BA spores to the LDLNs}

Active migration of particle or antigen-bearing LDCs to the LDLNs in both steady-state and inflamed conditions requires the LN-homing G-protein-coupled chemokine receptor, CCR7. ${ }^{9}$ Thus, we examined whether CCR7 deficiency prevented accumulation of spore + cells in the LDLNs following i.t. infection. Approximately $3 \times 10^{8}$ spores were administered to groups of control B6 and B6.CCR7 ${ }^{-1-}$ mice. At 6 h.p.i., the LDLNs were harvested and single-cell suspensions analyzed by flow cytometry. A similar frequency and number of spore + cells was seen in both groups of mice (Figure 2a,b ). Thus, CCR7 deficiency did not significantly affect spore trafficking to LDLNs at 6 h.p.i.

To confirm that LDC migration was defective in the BAinfected CCR7 ${ }^{-1-}$ mice, we repeated these studies by analyzing mice co-injected with a mixture (1:1) of FITC + latex beads and DyLight $649+$ spores. Beads or influenza virus entering the lungs are known to be carried to the LDLNs by LDCs, and this trafficking peaks $24 \mathrm{~h}$ after antigen exposure. ${ }^{9,16}$ Hence, we analyzed trafficking of spores and beads at $24 \mathrm{~h}$ after administering the mixed inocula i.t. By this time point, both bead + and spore + cell populations were readily detected in suspensions of the LDLNs from B6 mice (Figure 2c). The beads and spores largely segregated into different cell populations, with very few cells staining doubly for FITC and DyLight 649 (Figure 2c). As at 6 h.p.i., the frequencies of spore + cells in the LDLNs of B6 and B6.CCR7 ${ }^{-1-}$ mice were nearly identical at $24 \mathrm{~h}$ (Figure $2 \mathrm{c}$ ). In addition, the total number of spore + cells seen in the LDLNs of the CCR7 ${ }^{-1-}$ mice at $24 \mathrm{~h}$ was also not significantly reduced (Figure $\mathbf{2 d}$ ). There was a modest trend toward reduced numbers of spore + cells in the CCR7 $7^{-1-}$ LDLNs, which may indicate a minor contribution of the LDCs to BA spore traffic at this latter time point. However, bead + cells were present only in the LDLNs of B6 but not B6.CCR7 ${ }^{-1-}$ mice (Figure 2c,d). These data confirmed that trafficking of latex beads requires CCR7 and showed that BA spore trafficking was largely independent of CCR7 until at least 24 h.p.i.

\section{B cells are the primary cell type associated with BA spores in the LDLNs at early times after infection}

Given the initial trafficking of BA spores was largely CCR7 independent, we asked if a cell population other than LDCs might be responsible for the initial wave of BA spore dissemination. Consistent with the traffic of $\mathrm{BA}$ spores by professional antigenpresenting cells, spore + cells from LDLNs of A/J mice at 6 h.p.i. showed strong cell surface staining for class II major histocompatibility complex (MHCII) antigens (Figure 3a). Yet, very few of these spore + cells co-stained positive for the DC marker CD11c (Figure 3a,b). These results suggested that most of the spore + cells were not LDCs or that uptake of spores might cause LDCs to downregulate cell surface CD11c. Three lines of evidence excluded the latter explanation: (1) cell surface CD11c expression was similar in spore-positive and spore-negative LDCs at $6 \mathrm{~h}$ after in vivo infection (Figure 3c); (2) addition of BA spores to 

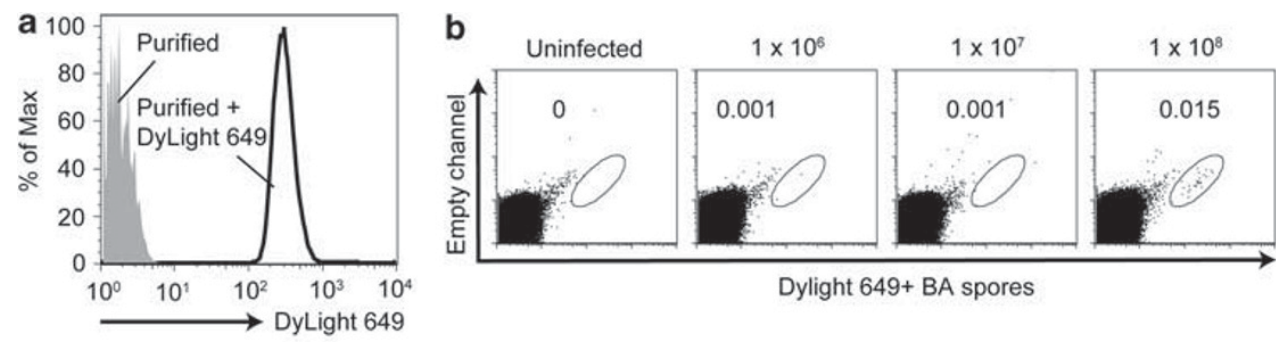

Dylight $649+$ BA spores
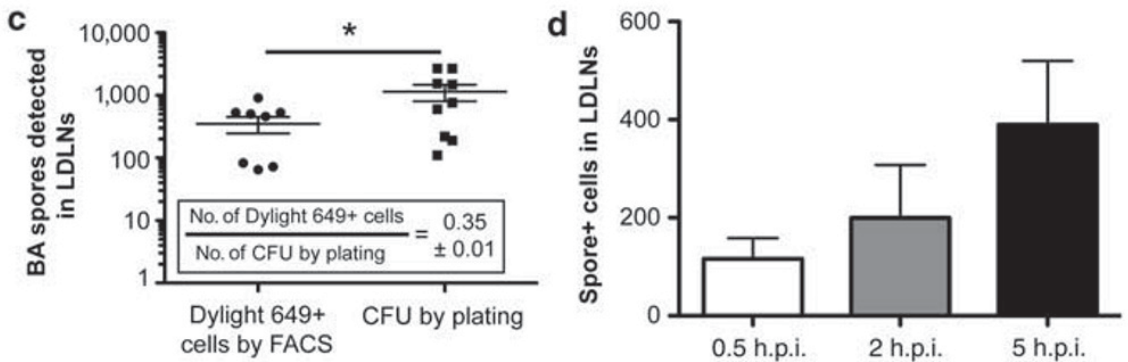

Figure 1 Labeled Bacillus anthracis (BA) spores are transported to the lung-draining lymph nodes (LDLNs) early after intratracheal (i.t.) infection. (a) Representative histogram demonstrating uniform labeling of BA spores with DyLight 649. Data were collected on an LSRII. Histogram shows labeled BA spores (black) compared with unlabeled BA spores (solid gray). DyLight 649-labeled spores were always checked for uniform labeling before using for an experiment. (b) Mice were infected i.t. with $1 \times 10^{6}, 1 \times 10^{7}$, or $1 \times 10^{8}$ BA spores or given phosphate-buffered saline (PBS) alone. LDLNs were analyzed by fluorescence-activated cell sorting (FACS) at 6 h.p.i. Fluorescence from BA spores is plotted against an empty channel to show percentage of spore + cells in the LDLNs. (c) LDLNs of infected A/J mice were analyzed for spore + cells by FACS and plated for heat-resistant colony-forming units (CFUs). (d) A/J mice were given $1 \times 10^{8} \mathrm{BA}$ spores i.t. and single-cell suspensions from LDLNs were analyzed by flow cytometry at $30 \mathrm{~min}$, and 2 and $5 \mathrm{~h}$ after infection for total number of spore + cells. Data in panels $\mathbf{c}$ are pooled from three independent experiments with $n=2-3$ per group. ${ }^{*} P<0.05$. Experiments in panels $\mathbf{b}$ and $\mathbf{d}$ are representative of two independent experiments with $n=2-3$ each.

bone marrow-derived DCs cultured in granulocyte-macrophage colony-stimulating factor did not alter CD11c expression (data not shown); and (3) on both a percentage and a numerical basis, over $3 / 4$ of the spore + cells isolated from LDLNs stained positive for the B-cell surface markers immunoglobulin M (IgM) and B220 (Figure 3a,b). BA spores also rapidly reached the LDLNs and associated with B cells following an intranasal (i.n.) route of infection, and when using green fluorescent protein (GFP)expressing or fixed spores (Supplementary Figure S1 online). Thus, spore association with B cells was not an artifact of germination or labeling. Moreover, DyLight 649+ BA spores were visible at the surface of B220 + cells sorted from the LDLNs of i.t.-infected mice (Figure 3d). Sorted spore + cells had large nuclei and the small cytoplasm typical of lymphocytes. Spore + cells from LDLNs of infected mice also expressed CD19, IgD, $\mathrm{CD} 21$, and CD23 (Figure 3e). B cells were also the predominant spore + cell type in the LDLNs of B6.CCR7 ${ }^{-1-}$ mice (Figure 3f). These data confirmed that the $\mathrm{B}$ cells are the antigen-presenting cell type predominantly associated with BA spores in the LDLNs early after i.t. or i.n. infection, and suggested that $B$ cells might play an important role in the early dissemination of BA spores.

\section{BA spores associate with both LDCs and B cells soon after entering the lungs}

We next asked whether BA spores associate with B cells in the lungs before trafficking to the LDLNs. Not surprisingly, we found that AMs and LDCs were the major spore + cell types recovered from single-cell suspensions of collagenase-digested infected lungs (Figure 4a). However, BA spores were also associated with $\mathrm{B}$ cells in these lung digests. Roughly $10^{4}(\sim 1 \%)$ of the $\sim 10^{6}$ recovered B cells stained positive for BA spores (Figure 4a). To further characterize spore + cells in situ, cryosections were prepared from infected lungs and stained using antibodies to IgM and CD11c (Figure $4 \mathbf{b}, \mathbf{c}$ ). In both uninfected and BA-infected lungs, IgM $+B$ cells were seen in proximity to both airway and alveolar epithelia (yellow arrows in Figure 4b). DyLight 649+ spores frequently colocalized with airway-lining epithelium and with the lung B cells and LDCs located beneath the epithelium by $1 \mathrm{~h}$ after pulmonary challenge (Figure $4 \mathrm{~b}, \mathrm{c}$ ). Thus, BA spores associated with lung $B$ cells before the observed association with $\mathrm{B}$ cells in the LDLNs. Moreover, such associations were readily observed in situ within lungs of infected mice.

B cells of diverse origin bind Bacillus spores independent of complement receptors, IgM expression, and BCR specificity

BA spores rapidly bound to $B$ cells when added to single-cell suspensions of cells from mouse spleens (Figure 5a), LNs, and lungs (Figure 5b). Indeed, in these cell culture experiments, which used suspensions from uninfected tissues, $\operatorname{IgM}+\mathrm{B} 220+$ $\mathrm{B}$ cells were the dominant cell type found to associate with the BA spores. These ex vivo cultures contained only heat-inactivated serum. Thus, the ability of BA spores to associate with lung B cells was not dependent on complement deposition on the BA spore surface.

Following their incubation with heat-inactivated mouse serum, the BA spores stained positive with an anti-IgM antibody 

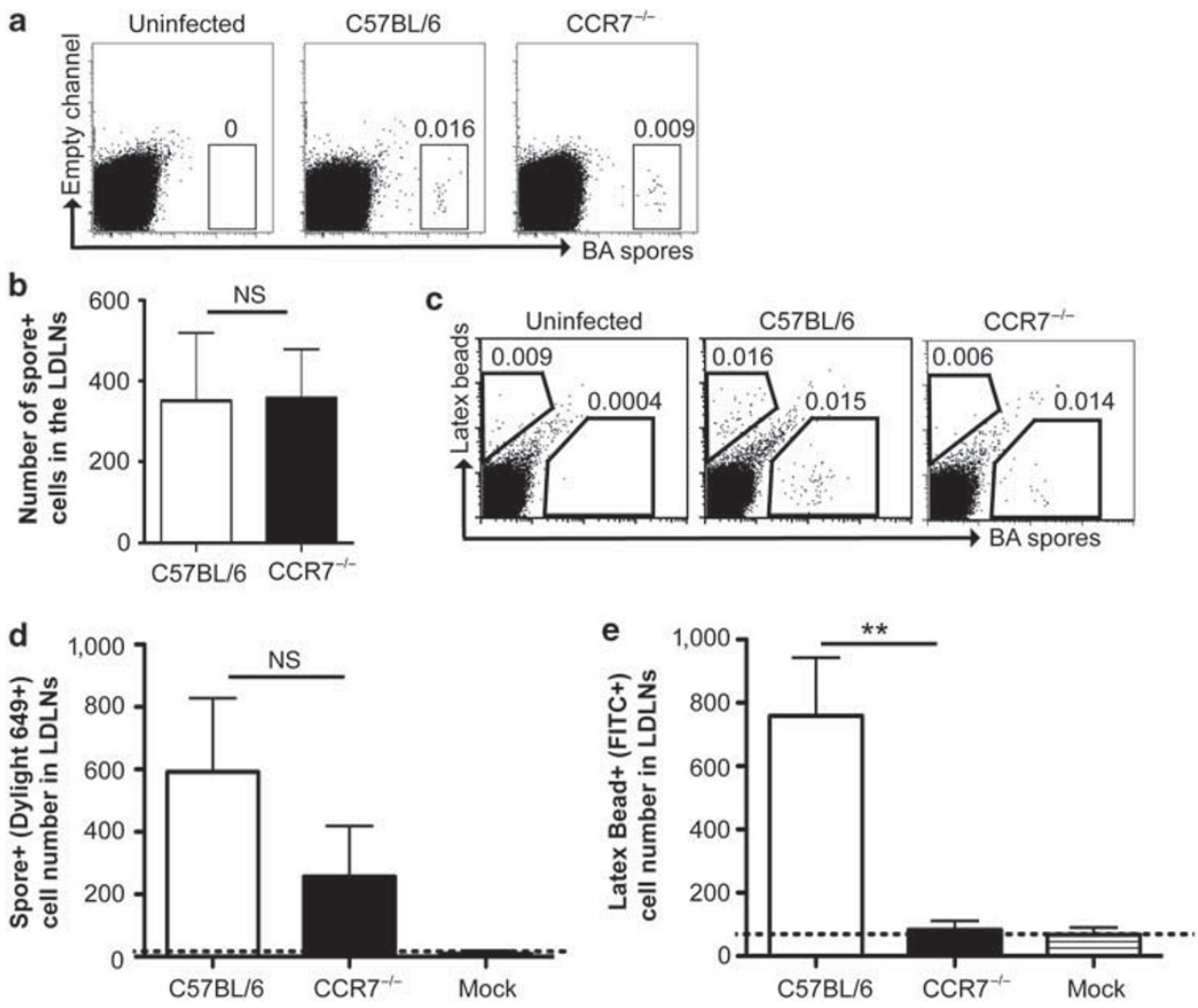

Figure 2 Rapid transport of Bacillus anthracis (BA) spores to the lung-draining lymph nodes (LDLNs) is independent of CCR7 (chemokine (C-C motif) receptor 7). (a) C57BL/6 ( $n=4)$ or B6.CCR7 ${ }^{-/-}(n=4)$ mice were infected intratracheally (i.t.) with $3 \times 10^{8}$ DyLight $649-$ labeled BA spores. LDLNs were analyzed for spore + cells at 6 hours post injection (h.p.i). Data from representative mice are plotted with gated frequencies. The $y$-axis represents an empty channel. (b) Calculated mean number of spore + cells from each group in panel a. (c) C57BL/6 $(n=3)$ or B6.CCR7 ${ }^{-1-}(n=4)$ mice were inoculated i.t. with a mixture of $1 \times 10^{8} \mathrm{BA}$ spores and $1 \times 10^{8} \mathrm{FITC}+$ latex beads. LDLNs were analyzed by flow cytometry at $24 \mathrm{~h}$.p.i. Spore $+(x$-axis $)$ and bead + ( $y$-axis) cells from representative mice are plotted with gated frequencies. Data in panels a-c are representative of two independent experiments. (d) Calculated mean number of spore+ (DyLight 649+) cells from each group in panel c. (e) Calculated mean number of Latex bead (FITC+) cells from each group in panel $\mathbf{c}$. Data in panels $\mathbf{d}$ and $\mathbf{e}$ are pooled from two independent experiments with $n=3-4$ per group. ${ }^{* *} P<0.005$; NS, not significant.

(Figure 5c), suggesting they might interact with $\operatorname{IgM}$ on the B-cell surface. This finding was consistent with a prior study that showed single-chain IgM fragments bound with high affinity to BA spores selectively when a $\mathrm{K}$ light chain was used in the antibody fragment. ${ }^{23}$ However, pretreatment of BA spores with heatinactivated mouse serum or purified mouse IgM failed to reduce the binding of BA spores to B cells in vitro (Figure $\mathbf{5 d}$ and data not shown). Moreover, spores also bound to IgM-negative A20 $\mathrm{B}$ cells (Figure 5e). Finally, the BA spores bound to intact B cells expressing Ig $\kappa+$ or Ig $\lambda+$ with a similar frequency (Figure $\mathbf{5} \mathbf{f}, \mathbf{g}$ ). Thus, the association of BA spores with intact B cells occurred independent of cell surface IgM and independent of light chain and BCR specificity. BA spores also bound immortalized human B-cell lines (Figure $5 \mathbf{h}$ ). Thus, the receptor for the BA spores on the $\mathrm{B}$-cell surface is conserved in humans and mice, but appears to be expressed by only a subset of B cells.

\section{Depletion of B cells significantly impairs BA spore traffic to LDLNs and increases survival of mice during a lethal pulmonary infection}

Although DCs traffic antigens from peripheral to lymphoid tissues, it is less clear whether B cells routinely traffic from the lung mucosa to LDLNs. To test whether B cells might traffic BA spores or other antigens from the lungs to the LDLNs, we isolated B cells from spleens of Ub-gfp mice and administered them i.t. into naive B6 mice. At $24 \mathrm{~h}$ after transfer, $1-2 \times 10^{6}$ could be recovered from lung digests and 100-200 gfp + B cells were detected in the LDLNs (Figure 6a). These data demonstrate that a proportion of the $\mathrm{B}$ cells present in the lung traffic to the LDLNs in the absence of any infectious stimuli. Such traffic appears to primarily occur via the lymphatics rather than the bloodstream, as we failed to detect gfp + B cells in the blood or nondraining (inguinal) LNs of the recipient mice (data not shown). In a related experiment, both wild-type and CCR7 ${ }^{-1-}$ $B$ cells (CD45.2) trafficked to LDLNs in congenic CD45.1 recipient mice (Figure 6b). This confirmed that $\mathrm{B}$ cells accessed the LDLNs independent of CCR7 expression.

We next evaluated how depletion of lung B cells before infection affected trafficking of BA spores to the LDLNs. To deplete $B$ cells, $A / J$ mice were given a single intraperitoneal injection of $250 \mu \mathrm{g}$ anti-CD20 or an isotype control antibody. An analysis of lung digests revealed extensive $\mathrm{B}$-cell depletion 7 to 8 days after the anti-CD20 treatment, with B-cell numbers in LDLNs and lungs reduced by $85-90 \%$ (Figure 6c). Thus, each organ 

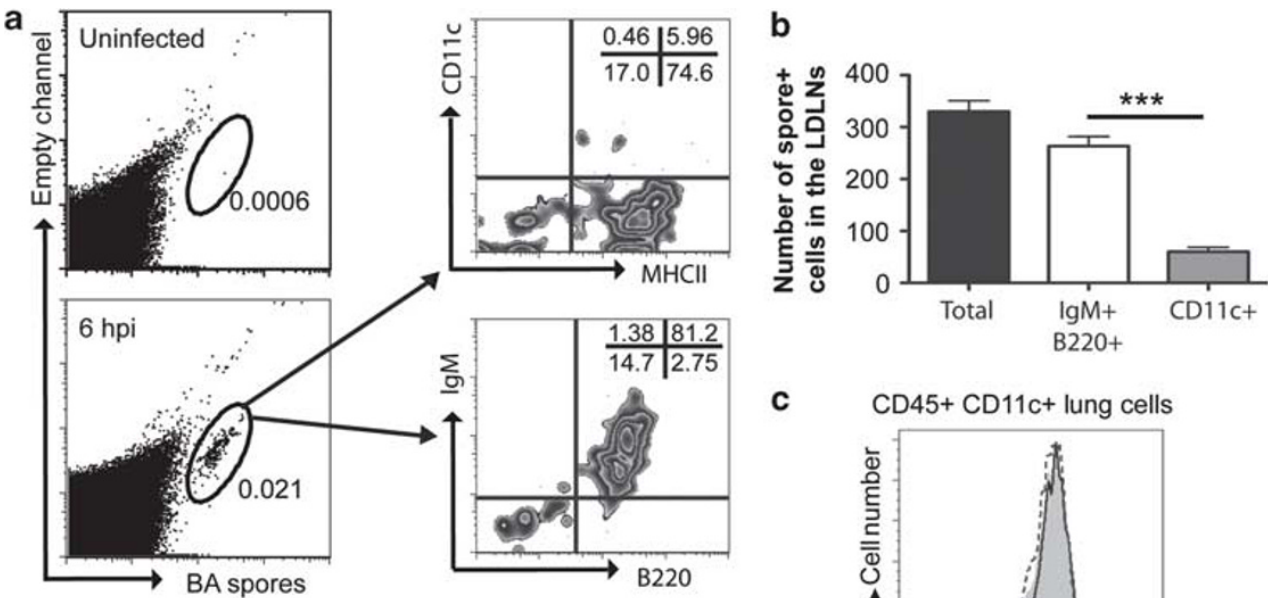

C CD45+ CD11c+ lung cells
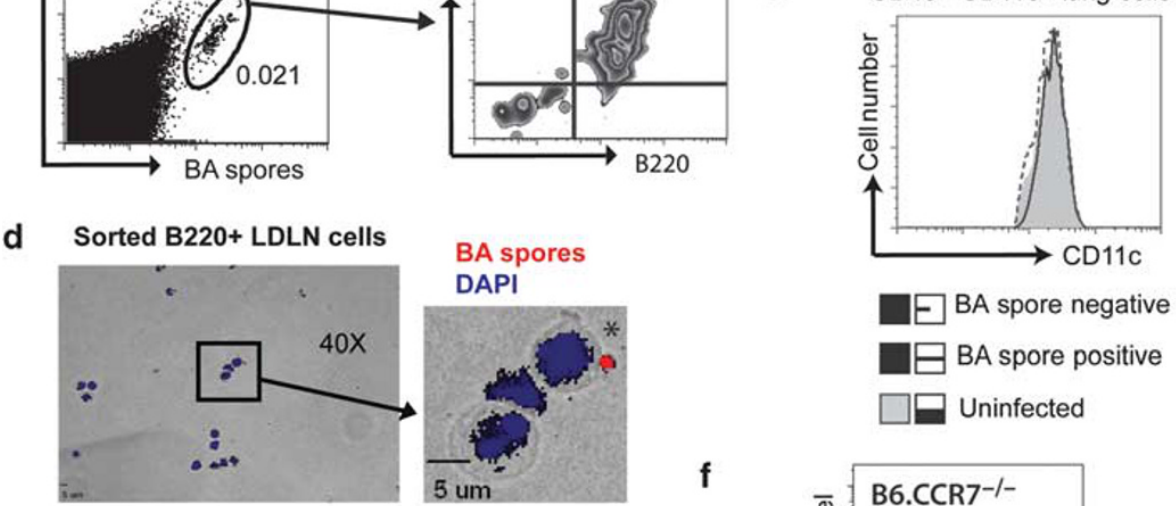

BA spore negative

$\square$ BA spore positive

Uninfected

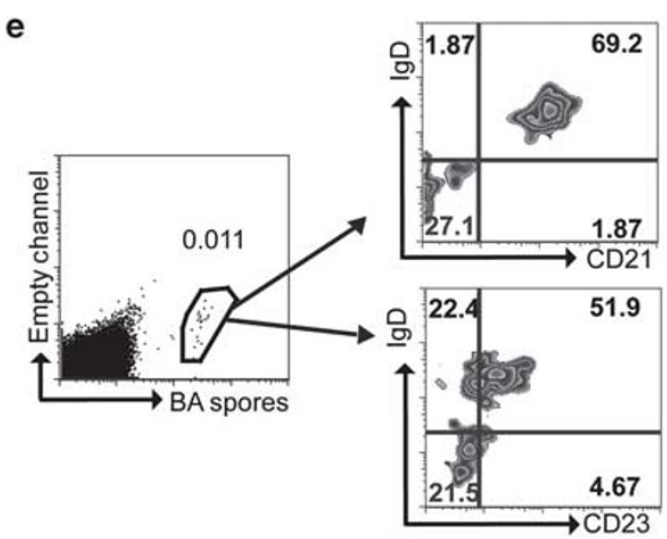

$\mathbf{f}$
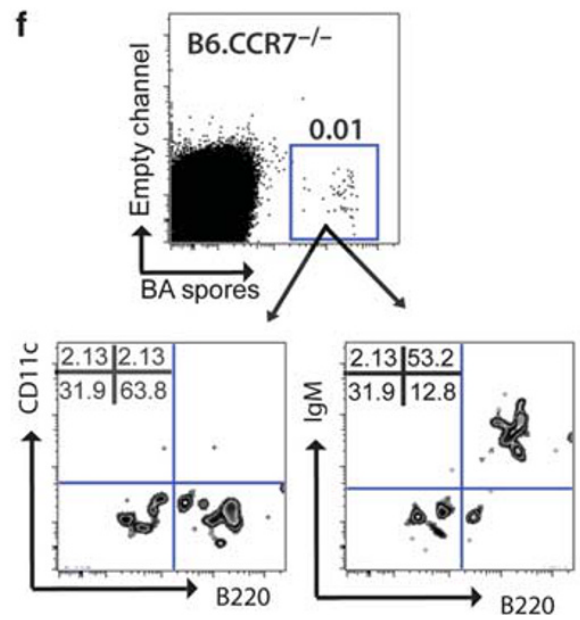

Figure 3 Bacillus anthracis (BA) spores that rapidly reach the lung-draining lymph nodes (LDLNs) are primarily associated with B cells. (a) At 6 hours post injection (h.p.i.) with $1 \times 10^{8} \mathrm{BA}$ spores intratracheal (i.t.), single-cell suspensions were pooled from LDLNs of A/J mice ( $\left.n=4\right)$ and analyzed by flow cytometry. Left panels show the gating strategy for spore + cells. Panels on the right depict the expression of B-cell markers (B220, immunoglobulin M (IgM), and class II major histocompatibility complex (MHCII)) and dendritic cell (DC) markers (CD11c and MHCII) on spore+ cells. (b) Quantification of spore + DCs and B cells from panel $\mathbf{a}$. Data in panels $\mathbf{a}$ and $\mathbf{b}$ are representative of three independent observations with $n=3$ per group. ${ }^{\star \star \star} P<0.0005$. (c) $\mathrm{A} / \mathrm{J}$ mice were infected i.t. with $10^{8} \mathrm{BA}$ spores. At 6 h.p.i., lungs were harvested and processed into a single-cell suspension. Lung cells were stained and CD11c expression levels were assessed on CD45+ CD11c + cells that were either DyLight 649+ (solid black line) or DyLight 649- (dashed line). Staining of CD11c on gated CD45 + CD11c+ cells from an uninfected mouse (filled gray histogram) is shown as a control (d) A/J mice $(n=4)$ were infected with $1 \times 10^{8}$ DyLight 649 -labeled BA spores intranasally (i.n.). At $12 \mathrm{~h}$.p.i., single-cell suspensions of the LDLNs were pooled and B220 + cells were positively sorted using MACS columns. Sorted cells were cytospun onto glass slides and stained for 4,6-diamidino2-phenylindole (DAPI). Shown is a representative merged image of spores (red) and nuclei (blue) using bright field and fluorescence. (e) A/J mice were infected with $1 \times 10^{8}$ fluorescent BA spores i.t. LDLNs were harvested at 6 h.p.i. and single-cell suspensions were analyzed for the expression of B-cell markers IgD, CD21, and CD23 (bottom panels) on gated spore + cells. (f) Gated, spore + cells from LDLNs of B6 and B6.CCR7 ${ }^{-/-}$mice were analyzed for DC and B-cell surface markers. Data in panel $\mathbf{c}$ are representative of three independent experiments. (d-f) Results from two independent experiments with $n=3-4$ per group are shown.

contained only $\sim 1-2 \times 10^{5}$ residual B cells. Mice were infected on day 8 with an i.t. dose of $1 \times 10^{8}$ unlabeled BA spores. At 6 h.p.i., LDLNs were harvested and lysates were plated to determine the number of heat-resistant CFUs (spores). Despite considerable variation in the recovery of CFUs from LDLNs of individual mice, the results indicated that B-cell depletion significantly impaired transport of viable spores to the LDLNs (Figure 6d). The average number of spores trafficked to LDLNs was also reduced from $\sim 4,500$ to $\sim 600$ in two experiments comparing $\mathrm{BALB} / \mathrm{c}$ mice and B-cell-deficient mb-1-null mice (Figure 6e). 
a
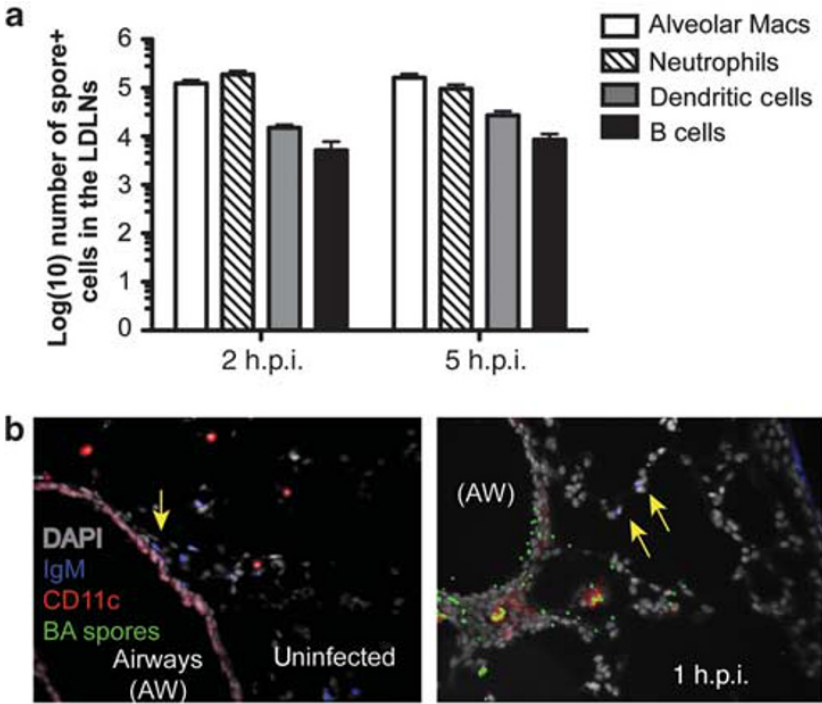

C

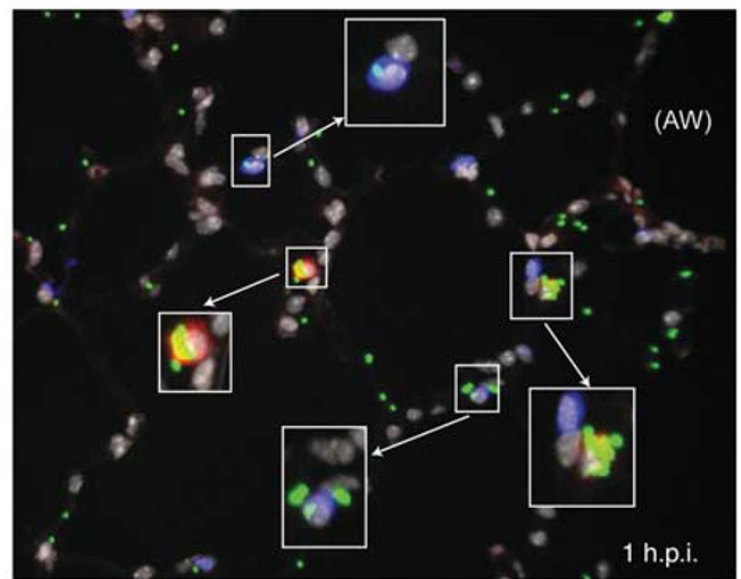

Figure 4 Bacillus anthracis (BA) spores rapidly associate with $B$ cells in the lungs of infected mice. (a) $A / J$ mice were infected intratracheally (i.t.) with $1 \times 10^{8}$ DyLight 649 -labeled BA spores. At 2 and 5 hours post injection (h.p.i.), bronchoalveolar lavage (BAL) was performed. Lavaged lungs were perfused, excised, and digested with collagenase to prepare single-cell suspensions. Lung digests were stained and analyzed by fluorescence-activated cell sorting (FACS). CD45+ cells were selected from the live gate and analyzed for cell populations as previously reported, ${ }^{30}$ including B cells (IgM + CD19+), alveolar macrophages (highly autofluorescent, CD11 C+, side scatter ${ }^{\text {hi }}$ and $\mathrm{MHCII}^{\mathrm{lo}}$ ), lung dendritic cells (CD11C+, SSClo, MHCll + ), and neutrophils $(\mathrm{Ly} 6 \mathrm{G}+)$. The number of each gated population that was spore + was calculated and graphed (mean \pm S.e.m.). Data are representative of three independent experiments with $n=3$ per group. (b, c) Cryosections of lower right lobes from lungs of naive (left panel) and infected (right panel) mice were analyzed for the distribution of BA spores (green), immunoglobulin M (IgM; blue), CD11c (red), and 4,6-diamidino-2-phenylindole (DAPI; gray). Airways (AW) are labeled and insets show spore association with either IgM + cells or CD11 + cells. Arrows in panel b highlight B-cell locations. All images were taken at original magnification $\times 25$. Similar observations were made in two independent experiments with $n=2-3$ per group.

In contrast, B-cell depletion had no effect on the total numbers of CFUs recovered from lungs of the infected mice (Figure 6f). B-cell depletion did not significantly alter the numbers of CD11b + Ly6G + neutrophils, CD11c + SigF + MHCII-
SSChi AMs, CD11c + MHCII + SSClo LDCs, CD4 + or CD8 + $\mathrm{T}$ cells, or Ly6G-Ly6C $+\mathrm{CD} 11 \mathrm{~b}+$ monocytes recovered from digests of BA-infected animals (data not shown). Thus, the presence of $B$ cells had no effect on the numbers of live spores in the lungs or on the numbers of phagocytes or other immune cells available to capture spores in the lungs, yet B cells increased traffic of live spores to the LDLNs.

Using Sterne-susceptible A/J mice, we next investigated the effects of B-cell depletion on susceptibility to lethal BA infection (Figure 7). Challenge of control mice with $2-3 \times 10^{6}$ purified, unlabeled BA spores caused $100 \%$ lethality within $65 \mathrm{~h}$ of infection. In contrast, $20 \%$ of the mice depleted of $B$ cells remained alive at this time point (Figure $7 \mathbf{b}$ ). In three independent survival experiments, the median time to death for mice receiving this dose of BA spores was significantly higher for B-cell depleted vs. control mice ( 59 vs. $48 \mathrm{~h}$ ). When a lower dose $\left(10^{5}\right)$ of BA spores was given, the mice depleted of B cells also showed an increased survival rate ( $\sim 40 \%$ vs. $20 \%$ in Figure 7c). B-cell depletion also increased the mean survival time from $\sim 3$ to $\sim 9$ days in these experiments. Thus, depletion of $B$ cells significantly delayed the progression of inhalation anthrax and improved survival following lung exposure to BA spores.

\section{DISCUSSION}

Our studies have revealed novel aspects of lung B-cell biology and a novel role for $\mathrm{B}$ cells during lung infection by BA. We found that a subset of $B$ cells from lungs and other tissues are capable of binding BA spores with strong affinity or avidity. In addition, we found that some of the $B$ cells present in the lung can traffic to the LDLNs. Thus, when BA spores interact with lung $B$ cells following i.t. or i.n. spore inoculation, they are rapidly transported to the LDLNs. Reductions in the numbers of lung and LDLN B cells reduced this rapid spore transport to the LDLNs and significantly delayed death of $\mathrm{A} / \mathrm{J}$ mice from a lethal inhalation anthrax infection using the attenuated Sterne strain. These results suggest that early, B-cell-dependent traffic of BA spores from the lung mucosa to the LDLNs (or possibly other sites) exacerbates host susceptibility to BA by providing this pathogen an early foothold to establish lethal systemic infection.

The rapid transport of BA spores from the lungs to the LDLNs was first observed over 50 years ago. ${ }^{11}$ Yet, the mechanisms for such transport have remained enigmatic. Consistent with findings from other infection models $, 2,5$ prior data indicated that LDCs associate with BA spores in the lungs and thus might promote their dissemination. ${ }^{3}$ However, trafficking of LDCs to the LDLNs requires CCR7, ${ }^{9}$ and we observed equivalent BA spore traffic from lungs to LDLNs in wild-type and CCR7-deficient mice. Using the minimal doses of labeled spores that permitted reproducible detection of in vivo spore transport using flow cytometry $\left(\sim 10^{8}\right)$, we also found that the spores accumulating in the LDLNs early after infection were almost exclusively associated with B cells. As both DCs and B cells in the lung efficiently bind the labeled spores, this result indicates enrichment for spore $+B$ cells in the lungs. We considered whether spores 
might reach the LDLNs through passive transport in the lymph and then bind B or other LDLN cells in situ. However, $1 \mu \mathrm{m}$ particles cannot access draining LNs without the aid of migratory cells and BA spores are larger than this $(0.86 \times 1.4 \mu \mathrm{m}) .{ }^{24-26}$ Moreover, we failed to observe CCR7-independent transport of even $0.5 \mu \mathrm{m}$ latex beads to the LDLNs when given alone or co-inoculated with BA spores. Instead, we observed constitutive traffic of GFP + wild-type B cells and CD45.1-wild-type and CCR7 ${ }^{-1-}$ B cells from the lung to the LDLNs and found that depletion or genetic ablation of $B$ cells significantly reduced early spore dissemination to the LDLNs. These data support the interpretation that B cells carry spores to the LDLNs in a CCR7independent manner. We do not yet know why only a subset of the total B-cell population seems competent for spore binding. Although spore binding to IgM was observed, B cells also bound to spores independently of IgM. This IgM-independent binding may be due to expression of a unique spore-binding protein or lectin by a thus far undefined B-cell subset.

We also observed that B-cell depletion partially ameliorated disease progression in Sterne-susceptible A/J mice. The effects of B-cell depletion on host susceptibility were seen with inocula as low as $10^{5}$ spores, and were not attributable to reduced spore uptake in the lungs, or an altered recruitment of inflammatory cells in the lungs. Moreover, accumulation of spores in the LDLNs of the B-cell-depleted mice was significantly reduced. These data suggest that the presence of $B$ cells exacerbates lethal BA infection because of their ability to promote rapid dissemination of BA spores from the lung to distal tissues. It is not clear why depletion of B cells failed to completely prevent spore trafficking to the LDLNs and failed to more completely block death of A/J mice challenged with Sterne strain BA spores. However, the residual B-cell populations in the lungs and LDLNs may be responsible for this residual spore dissemination, and/or such dissemination may be mediated by other means. For example, the small population of spore + non-B cells observed in our studies (some of which were CD11c+) may traffic sufficient spores to establish a lethal systemic infection in these susceptible mice.

Recently, CCR7 expression was also found to be dispensable for systemic dissemination of the bacterial pathogens Yersinia and Salmonella from lung and intestinal mucosae. ${ }^{4,5,27}$ In one of these studies, it was reported that systemic dissemination of
Yersinia pseudotuberculosis involved multiple waves of pathogen traffic from the intestine, the first of which was not seen in mice lacking $\mathrm{B}$ cells. ${ }^{27}$ The absence of $\mathrm{B}$ cells was also previously shown to delay dissemination of $M$. tuberculosis from the lungs. ${ }^{20}$ The role played by B cells in these other models was not determined. However, in light of our data, these findings suggest that mucosal B cells might directly traffic these bacterial pathogens to lymphoid tissues. B cells might also conceivably deliver pathogens to other, non-lymphoid systemic tissues. However, in our studies we failed to observe GFP + B cells, spore + cells, or BA CFUs in the blood or other organs within the first 24 h.p.i.

In summary, our findings have revealed a previously unappreciated role for $B$ cells in a mouse model of inhalation anthrax. Our data suggest that B cells participate directly in the trafficking of BA spores to lymphoid tissues and thereby hasten the systemic dissemination of anthrax bacteria. As we also found that BA spores bound to human B cells, it is possible that $B$ cells also promote pathogen dissemination in humans. Moreover, the data from our studies suggest that, like DCs, $\mathrm{B}$ cells may routinely participate in antigen transport from mucosal to lymphatic tissues. A refined understanding of how $B$ cells promote pathogen/antigen trafficking may reveal new therapies to delay progression of inhalation anthrax and other diseases.

\section{METHODS}

Cell lines. Namalwa, A20, and Ramos B cells were from Drs James Hagman and Jing Wang (National Jewish Health, Denver, CO).

Mice. C57BL/6, BALB/c, A/J, B6.CCR7-/- , and B6.CD45.2+ mice, 6 to 8 weeks old, were purchased from Jackson Laboratories (Bar Harbor, ME). BALB.mb-1-null mice were from Dr Roberta Pelanda (National Jewish Health). Animals were housed in the National Jewish Health Biological Resource Center under veterinary care. All studies were approved by the National Jewish Health Institutional Animal Care and Use Committee and Institutional Biosafety Committee.

BA spores. The BA strain $34 \mathrm{~F} 2\left(\mathrm{pXO} 1^{+}, \mathrm{pXO} 2^{-}\right)$was obtained from the Colorado Serum Company (Denver, CO). GFP expressing B. anthracis (7702; GFP-BA) was a kind gift from Dr Timothy Hoover (US Army Medical Research Institute of Infectious Diseases (USAMRIID, Frederick, MD)). Sporulation was induced in Difco Sporulation Medium (BD Biosciences, Franklin Lakes, NJ). Cultures were centrifuged to pellet

Figure 5 Bacillus anthracis (BA) spores bind mouse and human B cells independent of complement, immunoglobulin M (IgM), and B-cell receptor (BCR) specificity. (a) Single-cell suspensions from spleens of naïve A/J mice were co-incubated with DyLight 649-labeled BA spores (multiplicity of infection $(\mathrm{MOI})=5)$ for $30 \mathrm{~min}$ at $37^{\circ} \mathrm{C}$ in a tissue culture dish. Cells were washed and stained for $\mathrm{B}$ cells $(\mathrm{IgM}+\mathrm{B} 220+)$, neutrophils/PMNs $(\mathrm{Ly} 6 \mathrm{G}+\mathrm{CD} 11 \mathrm{~b}+)$, macrophages $(\mathrm{CD} 11 \mathrm{~b}+)$, and dendritic cells (DCs; Cd11c +$)$. (b) Cells from the lungs and lymph nodes of naïve mice were co-incubated with BA spores and analyzed for B cells, neutrophils, and DCs+ Macs as in panel a. Ly6G-CD11b+ and Ly6G-CD11c+ populations are pooled here and percentages of respective cell types associated with DyLight $649+$ BA spores are shown. Data in panels $\mathbf{a}$ and $\mathbf{b}$ were pooled from four and two independent experiments respectively. (c) $1 \times 10^{8} \mathrm{BA}$ spores were incubated with phosphate-buffered saline (PBS) or $100 \mu \mathrm{l}$ of serum from a naïve C57BL/6 mouse for $1 \mathrm{~h}$ at $4^{\circ} \mathrm{C}$ and then washed and stained with Alexa488 conjugated anti-mouse $\operatorname{IgM}\left(\mathrm{F}_{\text {ab }}\right)(\mathrm{Jackson} \operatorname{ImmunoResearch})$. Histograms show IgM staining on BA spores. (d) BA spores $\left(1 \times 10^{8}\right)$ were incubated were $100 \mu$ l of heat-inactivated serum $\left(30 \mathrm{~min}\right.$ at $\left.56^{\circ} \mathrm{C}\right)$ from naive C57BL/6 mice for $1 \mathrm{~h}$ at $4^{\circ} \mathrm{C}$. (e) Mouse B-cell line, A20, was co-incubated with BA spores as in panel d and analyzed. Numbers in dot plots indicate percentage of total cells gated positive for BA spores. (f) Splenocytes from naïve A/J and C57BL/6 were co-incubated with BA spores at MOI=5 for $30 \mathrm{~min}$ at $37^{\circ} \mathrm{C}$ and stained for $\mathrm{B} 220, \mathrm{CD} 3, \lg \kappa$, and $\lg \lambda$. Shown is the gating strategy to identify CD3-B220 + B cells expressing $\lg \kappa$ and $\lg \lambda$ light chains. (g) Plots indicate percentages of $\lg \kappa+$ or $\lg \lambda+B$ cells from A/J (left panels) and C57BL/6 (right panels) that bind BA spores. Gated as in panel f. (h) Human B-cell lines Ramos (right panel) and Namalwa (center panel) were co-incubated with DyLight 649-labeled BA spores as in panel $\mathbf{d}$ and analyzed by flow cytometry. Data in panels $\mathbf{d}-\mathbf{h}$ are representative of two independent experiments done in triplicate. 
spores when $>90 \%$ sporulation was achieved as verified under a light microscope. Pellets were washed $3 \times$ with cold $\mathrm{dH}_{2} 0$, heated to $70^{\circ} \mathrm{C}$ for $30 \mathrm{~min}$, and stored $4^{\circ} \mathrm{C}$ overnight before passage through Renografin (Renografin-60, Bracco Diagnostics, Princeton, NJ) as previously described. ${ }^{23}$ After labeling of purified spores with DyLight 649 (Thermo
Scientific, Waltham, MA) according to the manufacturer's protocol, they were washed 5 times with ice-cold water to remove excess dye and analyzed on an LSRII flow cytometer (BD Biosciences, Bedford, MA) to confirm efficient and uniform labeling before use in experiments. Pure spores were stored at $4^{\circ} \mathrm{C}$ for no more than 1 month before use. a

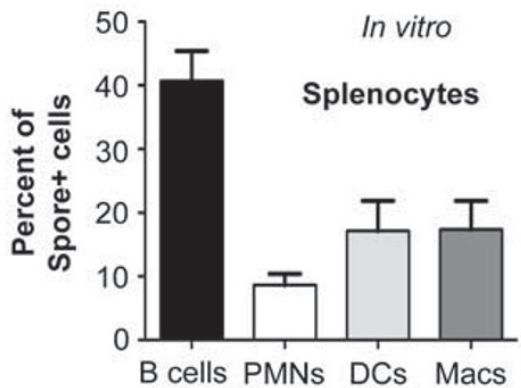

b

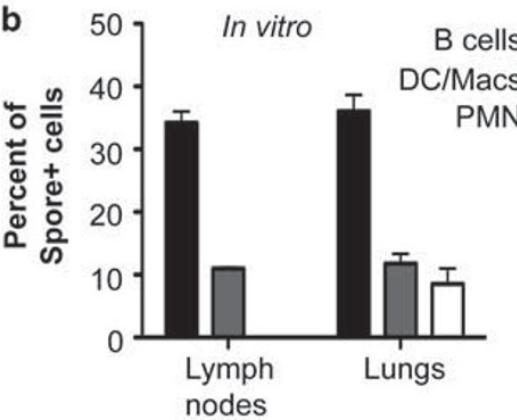

C

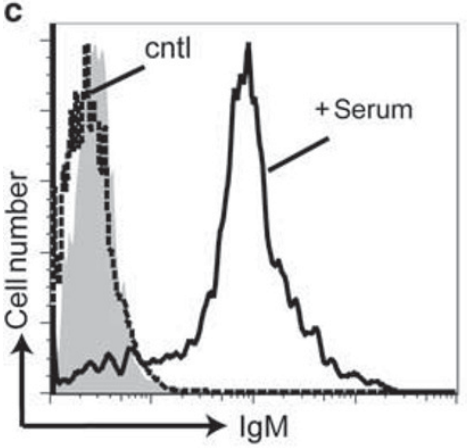

d Pretreatment

$$
\text { of spores }
$$

+ Heat-inactivated mouse serum
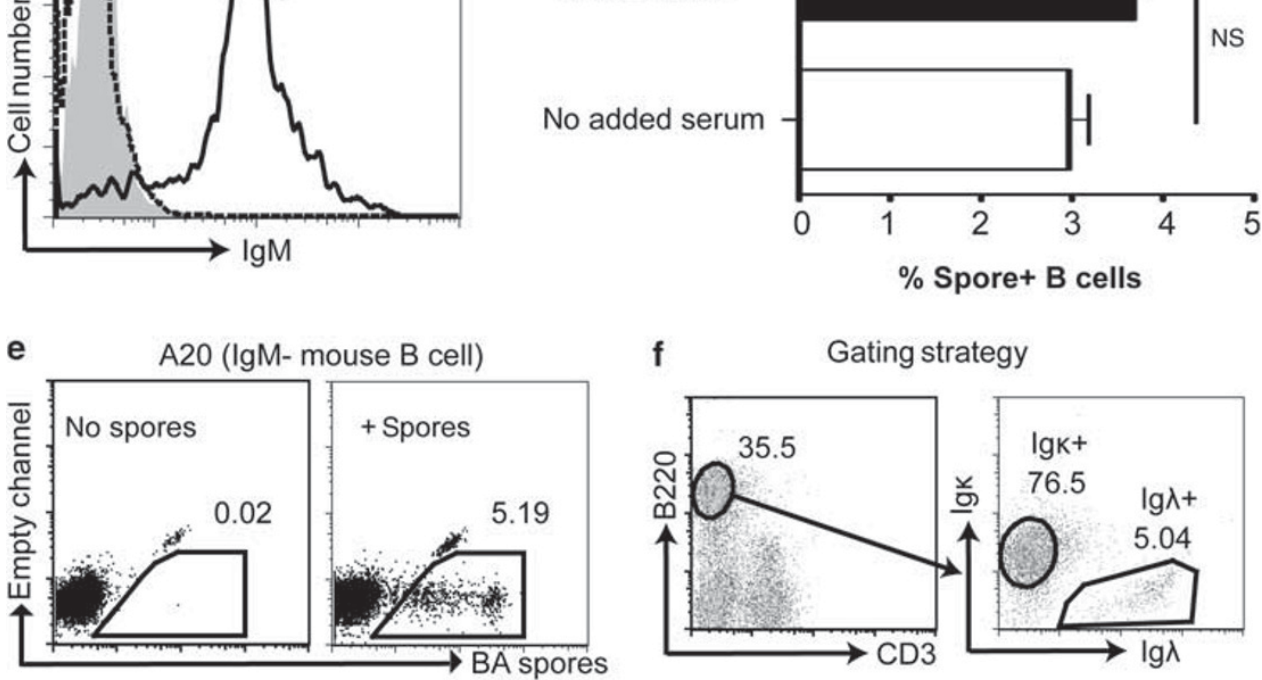

f Gating strategy

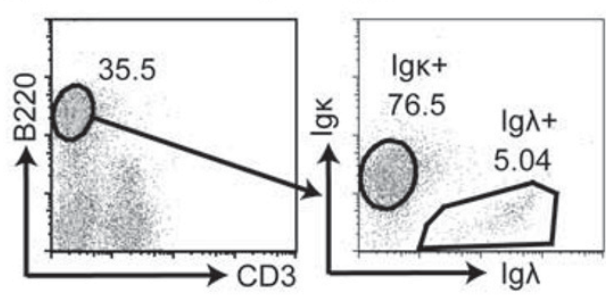

g
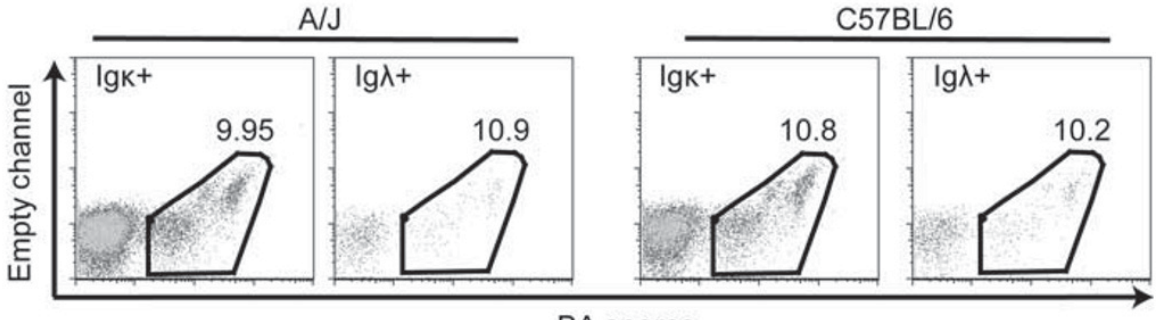

A spores

h BA spores -

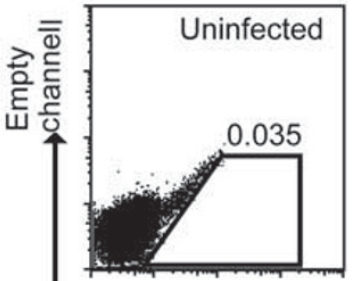

$+$
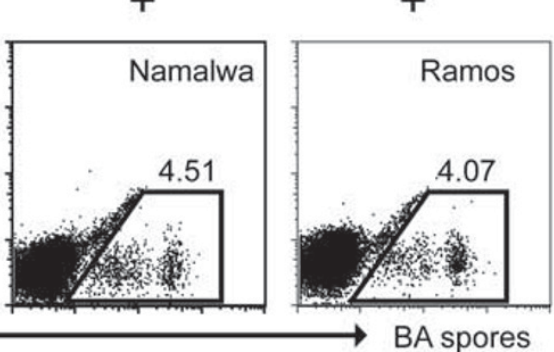


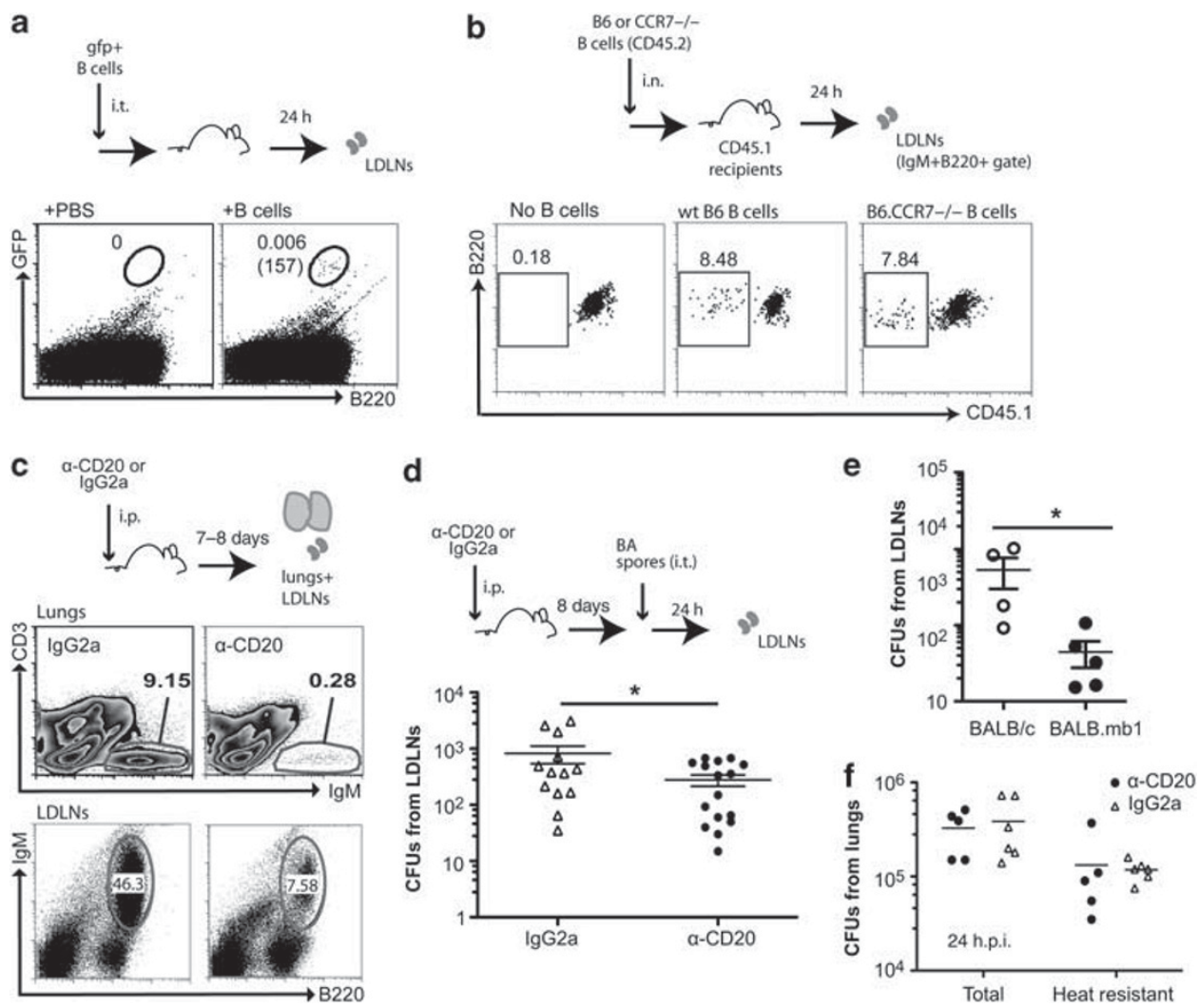

Figure 6 B cells traffic Bacillus anthracis (BA) spores to the lung-draining lymph nodes (LDLNs). (a) B cells from the spleen of C57BL/6-Ub-GFP mice were isolated using MACS columns by negative selection (>95\% CD43-). $15 \times 10^{6}$ of the purified CD43- cells were transferred intratracheally (i.t.) into each of three naive C57BL/6 recipients. LDLNs of recipients were analyzed at 24h.p.i. for expression of green fluorescent protein (GFP), B220, CD11c, and immunoglobulin M (IgM). Left panel shows B220 and GFP staining in control mice and right panel is a representative plot from a recipient mouse. Numbers represent percentage of total cells positive for GFP and B220 and numbers in parenthesis indicate the absolute number of cells in the gate. The gated GFP + cells were IgM + and CD11c-. Data representative of two independent experiments with $n=3$ per group.

(b) CD43 - cells were purified from age- and sex-matched wild-type (wt) B6 or B6.CCR7-/- mice (both CD45.2+ CD45.1-). $15 \times 10^{6}$ of the resulting B cells (92-94\% purity) were administered intranasally (i.n.) to congenic CD45.2+ B6 mice and LDLNs were harvested and analyzed $24 \mathrm{~h}$ later. Plots depict IgM + B220 + B cells that are gated to indicate the donor-derived (CD45.1+) population. Results are representative of two experiments using two recipient mice per group. (c) A/J mice were injected intraperitoneally (i.p.) with $250 \mu \mathrm{g}$ of $\alpha$-CD20 antibody or an isotype control IgG2a antibody. Lungs and LDLNs were analyzed for B cells on day 7. Plots show CD3 ( $y$-axis) and IgM ( $x$-axis) on live gated cells from lungs (top panels) or IgM ( $y$-axis) and B220 ( $x$-axis) staining on live LDLN cells. Numbers indicate percentage of cells in the gate. Dot plots are indicative of three independent experiments with $n=3$ per group. (d) A/J mice were i.p. injected with $\alpha$-CD20 antibody (Ab) or isotype control (IgG2a). On day 8, mice where challenged i.t. with $1 \times 10^{8}$ BA spores. At 6 h.p.i., LDLNs were harvested and processed into single-cell suspensions, and then lysed and heat treated at $70^{\circ} \mathrm{C}$ for 30 min to kill vegetative or geminated spores. Shown are numbers of recovered heat-resistant colony-forming units (CFUs) pooled from four independent experiments with $n=3-5$ mice per group. (e) BALB/c and BALB.mb1 mutant mice were infected i.t. with $2-3 \times 10^{6}$ BA spores. At 24 h.p.i., LDLNs were isolated and lysed and plated to determine the number of BA CFUs. Data are pooled from two independent experiments. (f) $A / J$ mice received an i.p. injection of $\alpha$-CD20 or isotype control (IgG2a). On day 8, mice were infected i.t. with $2-3 \times 10^{6}$ BA spores. At 24 h.p.i., homogenized lung lysates were plated for total and heat-resistant CFUs. Shown are data pooled from two independent experiments.

Bacterial infections and B-cell depletion. Mice were anesthetized with an intraperitoneal injection of xylazine $\left(2 \mathrm{mg} \mathrm{ml}^{-1}\right)$ plus ketamine $\left(10 \mathrm{mg} \mathrm{ml}^{-1}\right)$. The i.t injections were given in $50 \mu$ volumes using a laryngoscope as we previously detailed. ${ }^{22}$ For i.n. challenge, $30 \mu \mathrm{l}$ was pipetted into the left nostril and mice were held upright for 30 s to ensure delivery of full inoculum.

For B-cell depletion studies, $250 \mu \mathrm{g}$ of anti-CD20 antibody was injected intraperitoneally 7 days before infection. Purified anti-CD20 monoclonal (18B12; IgG2a) was a gift of Robert Dunn and Marilyn Kehry (Biogen Idec, Weston, MA). Purified IgG2a isotype control antibody (C1.18) was purchased from BioXCell (West Lebanon, $\mathrm{NH}$ ).

Flow cytometry. Bronchoalveolar lavage was performed as described. ${ }^{9}$ Where indicated, lungs were perfused with $10 \mathrm{ml}$ of ice-cold phosphatebuffered saline (PBS). Our protocol for obtaining lungs and LDLNs is described. ${ }^{22}$ The numbers of cells in LDLN analyses represent cells obtained from the same three LDLNs (two cranial mediastinal and one tracheaobronchial) per animal. For cell isolation, lungs were finely chopped and digested with $1 \mathrm{mg} \mathrm{m}^{-1}$ collagenase (Sigma, Ronkonkoma, $\mathrm{NY}$ ) for $30 \mathrm{~min}$ at $37^{\circ} \mathrm{C}$. EDTA was added to a final concentration of $10 \mathrm{mM}$, and then digest was passed through an $18 \mathrm{G}$ needle and $70 \mu \mathrm{m}$ cell strainer (BD Biosciences). LDLNs (two cranial mediastinal and one tracheaobronchial) were harvested from left thoracic cavity and digested $20 \mathrm{~min}$ with $1 \mathrm{ml}$ of $0.125 \%$ collagenase type IV (Worthington, Lakewood, $\mathrm{NJ}$ ) at $37^{\circ} \mathrm{C}$ in Hank's Balanced Salt Solution plus cations before strainer. Red bloods cells were lysed using ACK lysis buffer. Single-cell suspensions for flow cytometry were treated with anti-CD16/32 (2.4G2) to block Fc receptors and stained as described. ${ }^{28}$ The following antibodies were obtained: CD45 (30-F11), IgM (II/41), B220 (RA3-6B2), CD11c (N418), and CD11b (M1/70; all from eBioscience, San Diego, CA); Ly6G (1A8) 
a
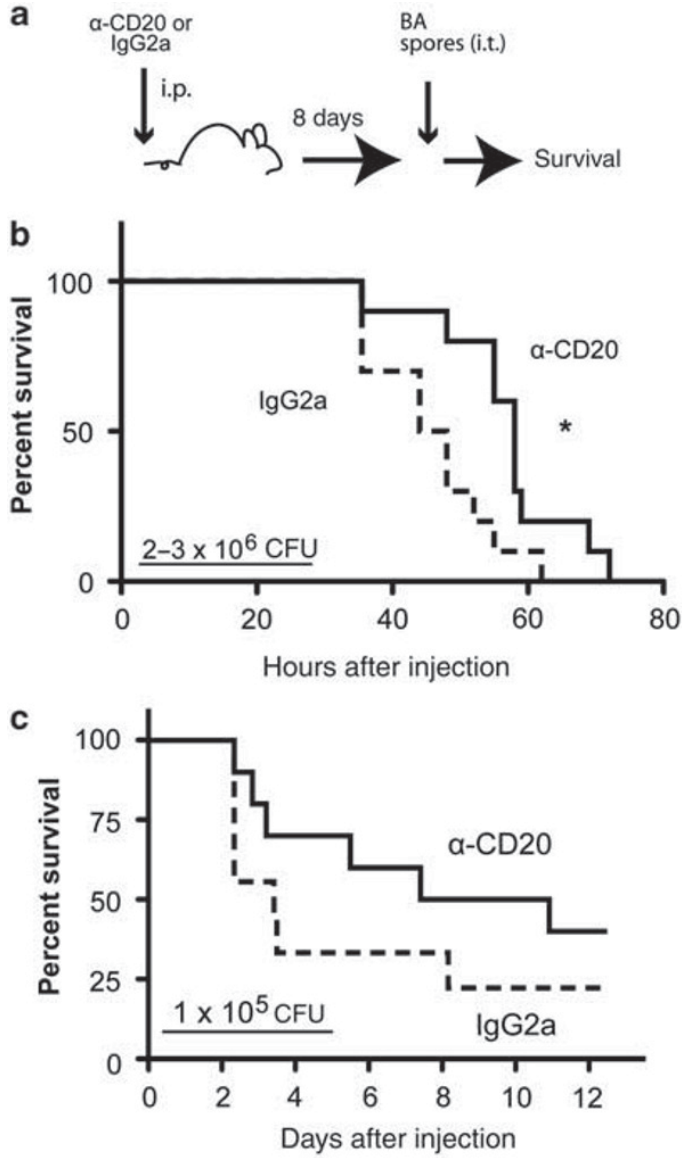

Figure 7 Depletion of $B$ cells delays death of $A / J$ mice following lethal infection of the lungs with the Sterne strain of Bacillus anthracis (BA). (a) A/J mice were injected intraperitoneally (i.p.) with $250 \mu \mathrm{g}$ of $\alpha$-CD20 or isotype control (IgG2a) antibody. At day 8 after injection, both groups were challenged with unlabeled BA spores and survival was monitored. (b) Survival of $\mathrm{A} / \mathrm{J}$ mice given $2-3 \times 10^{6} \mathrm{BA}$ spores. Data are representative of 3 independent experiments using 10 mice per group. (c) Survival of mice given $1 \times 10^{5}$ BA spores. Data are representative of 2 independent experiments using 10 mice per group. ${ }^{\star} P<0.05$.

and Igא (187.1; from BD Biosciences); MHCII (14-4-4S; from BioLegend, San Diego, CA); or Ig $\lambda$ (JC5-1; from Southern Biotech, Birmingham, AL). Samples fixed with $4 \%$ paraformaldehyde overnight were run on an LSRII (BD Biosciences) and analyzed using FlowJo software (Treestar, Ashland, OR).

CFU analysis. Lungs harvested into $10 \mathrm{ml}$ of $0.02 \% \mathrm{NP}-40$ were homogenized and incubated at room temperature for $15 \mathrm{~min}$ before plating serial dilutions. Single-cell suspensions of LDLNs prepared as above were pelleted, lysed in $500 \mu \mathrm{l} 0.02 \% \mathrm{NP}-40$, and incubated at room temperature for $15 \mathrm{~min}$ before plating. For heat-resistant CFUs, aliquots were incubated at $70^{\circ} \mathrm{C}$ for $30 \mathrm{~min}$ before plating.

Isolation of B cells and adoptive transfer. Splenic B cells were isolated by negative selection using anti-CD43 microbeads according to the manufacturer's protocol (Miltenyi Biotec, Auburn, CA). For adoptive transfers, $15 \times 10^{6} \mathrm{~B}$ cells were resuspended in PBS and injected i.t. or i.n. into anesthetized naïve mice.

Immunofluorescence microscopy of lung sections. Infected mice were killed and lungs were prepared as described elsewhere. ${ }^{29}$ Briefly, lungs were perfused with $10 \mathrm{ml}$ ice-cold PBS. The trachea was then cannulated and $1 \mathrm{ml}$ of optimum cutting temperature (OCT) compound freezing medium was injected into the lung. The trachea was tied to prevent leakage of OCT and lungs were harvested into ice-cold PBS. Lobes were separated and placed, convex side down, in plastic molds halffilled with OCT. Molds were transferred onto dry ice, filled with OCT, and stored at $-80^{\circ} \mathrm{C}$. Cryosections were cut on a cryostat and collected onto charged microscope slides (Thermo Scientific). Slides were stored at $-80^{\circ} \mathrm{C}$. Before staining, slides were air-dried at room temperature and rehydrated with PBS for $15 \mathrm{~min}$. Sections were blocked with anti-FcR (2.4G2) for $30 \mathrm{~min}$ and then stained with directly conjugated fluorophore-labeled antibodies specific for CD11c (N418) and Fab fragments specific for IgM ( $\mu$ chain specific; Jackson ImmunoResearch, West Grove, PA). After $1 \mathrm{~h}$ at room temperature, slides were washed $3 \times$ in PBS $(5 \mathrm{~min}$ each) and counterstained with 4,6-diamidino-2-phenylindole (DAPI). After three additional washes in PBS (5 min each), stained 5-7 $\mu \mathrm{m}$ cryosections were imaged using a Leica DMRXMA upright fluorescence microscope (Knowhill, UK). Images were analyzed using Slidebook 5.0 (3I, Atlanta, GA).

Cytospin. B cells from LDLNs of infected mice were enriched with antiB220 MACS microbeads (Miltenyi), cytocentrifuged onto glass slides, fixed with $4 \%$ paraformaldehyde, and stained for imaging and analysis as described above.

Statistical analysis. Data were processed and plotted in GraphPad PRISM (Graph-Pad Software, San Diego, CA). All error bars indicate s.e.m. Two-tailed unpaired $t$-tests were used for statistical analyses except for survival. For survival, curves were Kaplan-Meier, and $P$-values were calculated by Log-rank (Mantel-Cox) test. Throughout, ${ }^{\star} P<0.05$, ${ }^{* *} P<0.005$, and ${ }^{* * *} P<0.0005$.

SUPPLEMENTARY MATERIAL is linked to the online version of the paper at http://www.nature.com/mi

\section{ACKNOWLEDGMENTS}

We thank Peter Henson, John Cambier, Steven Dow, Raul Torres, and Elizabeth Redente for insightful scientific discussions, suggestions, and reagents. We are also grateful to Sue Reynolds, J. Wands, and Willi Born for assistance with cryosectioning and visualization of lung sections. This study was supported by a Department of Defense grant (W81XWH-071-0550) to L.L.L. and D.W.R. Additional support was from the NIH grant P01-Al022295.

\section{DISCLOSURE}

The authors declared no conflict of interest.

(c) 2012 Society for Mucosal Immunology

\section{REFERENCES}

1. Cossart, P. \& Sansonetti, P.J. Bacterial invasion: the paradigms of enteroinvasive pathogens. Science 304, 242-248 (2004).

2. Vazquez-Torres, A. et al. Extraintestinal dissemination of Salmonella by CD18-expressing phagocytes. Nature 401, 804-808 (1999).

3. Cleret, A. et al. Lung dendritic cells rapidly mediate anthrax spore entry through the pulmonary route. J. Immunol. 178, 7994-8001 (2007).

4. Bosio, C.M., Goodyear, A.W. \& Dow, S.W. Early interaction of Yersinia pestis with APCs in the lung. J. Immunol. 175, 6750-6756 (2005).

5. Voedisch, S. et al. Mesenteric lymph nodes confine dendritic cellmediated dissemination of Salmonella enterica serovar Typhimurium and limit systemic disease in mice. Infect. Immun. 77, 3170-3180 (2009).

6. Banchereau, J. \& Steinman, R.M. Dendritic cells and the control of immunity. Nature 392, 245-252 (1998).

7. Jang, M.H. et al. CCR7 is critically important for migration of dendritic cells in intestinal lamina propria to mesenteric lymph nodes. J. Immunol. 176, 803-810 (2006).

8. Ohl, L. et al. CCR7 governs skin dendritic cell migration under inflammatory and steady-state conditions. Immunity 21, 279-288 (2004). 


\section{ARTICLES}

9. Jakubzick, C., Tacke, F., Llodra, J., van Rooijen, N. \& Randolph, G.J. Modulation of dendritic cell trafficking to and from the airways. J. Immunol. 176, 3578-3584 (2006).

10. Forster, R. et al. CCR7 coordinates the primary immune response by establishing functional microenvironments in secondary lymphoid organs. Cell 99, 23-33 (1999).

11. Ross, J. The pathogenesis of anthrax following the administration of spores by the respiratory route. J. Pathol. Bacteriol. 73, 485-494 (1957).

12. Lincoln, R.E. et al. Role of the lymphatics in the pathogenesis of anthrax. J. Infect. Dis. 115, 481-494 (1965).

13. Lyons, C.R. et al. Murine model of pulmonary anthrax: kinetics of dissemination, histopathology, and mouse strain susceptibility. Infect. Immun. 72, 4801-4809 (2004).

14. Guidi-Rontani, C., Weber-Levy, M., Labruyere, E. \& Mock, M. Germination of Bacillus anthracis spores within alveolar macrophages. Mol. Microbiol. 31, 9-17 (1999).

15. Cleret, A., Quesnel-Hellmann, A., Mathieu, J., Vidal, D. \& Tournier, J.N. Resident $\mathrm{CD} 11 \mathrm{c}+$ lung cells are impaired by anthrax toxins after spore infection. J. Infect. Dis. 194, 86-94 (2006).

16. Legge, K.L. \& Braciale, T.J. Accelerated migration of respiratory dendritic cells to the regional lymph nodes is limited to the early phase of pulmonary infection. Immunity 18, 265-277 (2003).

17. Holt, P.G., Strickland, D.H., Wikstrom, M.E. \& Jahnsen, F.L. Regulation of immunological homeostasis in the respiratory tract. Nat. Rev. Immunol. 8, 142-152 (2008).

18. Kohlmeier, J.E. \& Woodland, D.L. Immunity to respiratory viruses. Annu. Rev. Immunol. 27, 61-82 (2009).

19. Gray, D., Gray, M. \& Barr, T. Innate responses of B cells. Eur. J. Immunol. 37, 3304-3310 (2007).

20. Bosio, C.M., Gardner, D. \& Elkins, K.L. Infection of B cell-deficient mice with CDC 1551, a clinical isolate of Mycobacterium tuberculosis: delay in dissemination and development of lung pathology. J. Immunol. 164, 6417-6425 (2000).

21. Neves, P. et al. Signaling via the MyD88 adaptor protein in B cells suppresses protective immunity during Salmonella typhimurium infection. Immunity 33, 777-790 (2010).

22. Rayamajhi, M. et al. Non-surgical intratracheal instillation of mice with analysis of lungs and lung draining lymph nodes by flow cytometry. J. Vis. Exp. pii, 2702 (2011).

23. Severson, K.M., Mallozzi, M., Driks, A. \& Knight, K.L. B cell development in GALT: role of bacterial superantigen-like molecules. J. Immunol. 184, 6782-6789 (2010).

24. Carrera, M., Zandomeni, R.O., Fitzgibbon, J. \& Sagripanti, J.L. Difference between the spore sizes of Bacillus anthracis and other Bacillus species. J. Appl. Microbiol. 102, 303-312 (2007).

25. Cyster, J.G. B cell follicles and antigen encounters of the third kind. Nat. Immunol. 11, 989-996 (2010).

26. Manolova, V. et al. Nanoparticles target distinct dendritic cell populations according to their size. Eur. J. Immunol. 38, 1404-1413 (2008).

27. Barnes, P.D., Bergman, M.A., Mecsas, J. \& Isberg, R.R. Yersinia pseudotuberculosis disseminates directly from a replicating bacterial pool in the intestine. J. Exp. Med. 203, 1591-1601 (2006).

28. Rayamajhi, M., Humann, J., Penheiter, K., Andreasen, K. \& Lenz, L.L. Induction of IFN-alphabeta enables Listeria monocytogenes to suppress macrophage activation by IFN-gamma. J. Exp. Med. 207, 327-337 (2010).

29. Wands, J.M. et al. Distribution and leukocyte contacts of gammadelta T cells in the lung. J. Leukoc. Biol. 78, 1086-1096 (2005).

30. Kim, T.S. \& Braciale, T.J. Respiratory dendritic cell subsets differ in their capacity to support the induction of virus-specific cytotoxic CD8+ T cell responses. PLoS One 4, e4204 (2009). 